\title{
Role of Tobramycin in the Induction and Maintenance of Viable but Non-Culturable Pseudomonas aeruginosa in an In Vitro Biofilm Model
}

\author{
Gianmarco Mangiaterra ${ }^{1, *(1)}$, Nicholas Cedraro ${ }^{1}$, Salvatore Vaiasicca ${ }^{1}$, Barbara Citterio ${ }^{2}$, \\ Roberta Galeazzi $^{1}{ }^{1}$, Emiliano Laudadio $^{3}{ }^{(0)}$, Giovanna Mobbili ${ }^{1}{ }^{(}$, Cristina Minnelli $^{1}{ }^{(0)}$, \\ Davide Bizzaro ${ }^{1}$ and Francesca Biavasco ${ }^{1}$ (D) \\ 1 Department of Life and Environmental Sciences, Polytechnic University of Marche, via Brecce Bianche, \\ 60131 Ancona, Italy; n.cedraro@pm.univpm.it (N.C.); s.vaiasicca@pm.univpm.it (S.V.); \\ r.galeazzi@staff.univpm.it (R.G.); g.mobbili@staff.univpm.it (G.M.); c.minnelli@pm.univpm.it (C.M.); \\ d.bizzaro@staff.univpm.it (D.B.); f.biavasco@staff.univpm.it (F.B.) \\ 2 Department of Biomolecular Science, Biotechnology Section, University of Urbino "Carlo Bo", \\ via Arco d'Augusto 2, 61032 Fano, Italy; barbara.citterio@uniurb.it \\ 3 Department of Materials, Environmental Sciences and Urban Planning, Polytechnic University of Marche, \\ via Brecce Bianche, 60131 Ancona, Italy; e.laudadio@staff.univpm.it \\ * Correspondence: g.mangiaterra@staff.univpm.it; Tel.: +39-071-220-4622; Fax: +39-071-220-4316
}

Academic Editors: Patrick Mester and Nicholas Dixon

Received: 5 May 2020; Accepted: 8 July 2020; Published: 10 July 2020

\begin{abstract}
The recurrence of Pseudomonas aeruginosa (PA) biofilm infections is a major issue in cystic fibrosis $(\mathrm{CF})$ patients. A pivotal role is played by the presence of antibiotic-unresponsive persisters and/or viable but non-culturable (VBNC) forms, whose development might be favored by subinhibitory antibiotic concentrations. The involvement of tobramycin and ciprofloxacin, widely used to treat $\mathrm{CF}$ PA lung infections, in the abundance of VBNC cells was investigated in PA biofilms models. In vitro biofilms of the laboratory strain PAO1-N and the clinical strain C24 were developed and starved by subculture for 170 days in a non-nutrient $(\mathrm{NN})$ broth, unsupplemented or supplemented with one-quarter minimal inhibitory concentration (MIC) of tobramycin or ciprofloxacin. VBNC cells abundance, estimated as the difference between total live (detected by qPCR and flow cytometry) and colony forming unit (CFU) counts, showed a strain- and drug-specific pattern. A greater and earlier abundance of VBNC PAO1-N cells was detected in all conditions. Exposure of the C24 strain to NN and NN + ciprofloxacin induced only a transient VBNC subpopulation, which was more abundant and stable until the end of the experiment in tobramycin-exposed biofilms. The same response to tobramycin was observed in the PAO1-N strain. These findings suggest that low tobramycin concentrations might contribute to PA infection recurrence by favoring the development of VBNC forms.
\end{abstract}

Keywords: cystic fibrosis; Pseudomonas aeruginosa biofilms; viable but non-culturable forms; tobramycin; recurrent infections

\section{Introduction}

Pseudomonas aeruginosa causes chronic infections [1], which are the main cause of death in cystic fibrosis (CF) patients. Biofilm production [2], multidrug resistance, the formation of pathogen reservoirs in different regions of the lung [3] and persistent, dormant bacterial forms [4] are key factors in infection resilience. Viable but non-culturable (VBNC) cells, a later stage of dormancy, do not grow on bacteriological media, and exposure to favorable conditions is not sufficient for the immediate recovery of full metabolic activity and culturability; for this to happen, conditions must include factors such as 
those protecting against oxidative stress [5]. VBNC cells have been demonstrated in CF sputum [6-8], being more abundant in patients showing chronic P. aeruginosa colonization and limited improvement in lung function after treatment [6]. Our group has documented VBNC cells in a study of clinical samples, where P. aeruginosa abundance was measured by routine culture-based methods and by a species-specific qPCR protocol, which was demonstrated to detect both culturable and non-culturable viable bacteria [8]. Although the factors involved in VBNC cell induction in the lungs of CF patients are still to be elucidated, their pulmonary environment is characterized by the presence of multiple stress factors that include low-level oxygen, catabolic waste, nutrient depletion, suboptimal $\mathrm{pH}$ as well as antibiotics, since chronic infections are repeatedly treated with drugs such as tobramycin and ciprofloxacin. Notably, the antibiotic may be found in subinhibitory concentrations in the biofilm matrix due either to its poor penetration or to its decrease between two different therapeutic cycles [9]. This is a cause of concern, particularly in strong biofilm-producing bacteria like P. aeruginosa. According to the hormesis theory, developed by Davies and co-workers, subinhibitory antibiotic concentrations may modulate bacterial gene expression [10]. We have previously demonstrated a role for vancomycin and quinupristin/dalfopristin in the maintenance of VBNC Staphylococcus aureus cells in biofilms cultured in nutrient-depleted medium [11]. According to a more recent study [12], culture in the presence of gentamicin resulted in a more robust induction of VBNC P. aeruginosa cells than in the presence of carbenicillin or colistin, suggesting a main role for the protein synthesis inhibitors. This work was undertaken to assess the ability of subinhibitory concentrations of tobramycin or ciprofloxacin-which are routinely used to treat CF patients with $P$. aeruginosa lung infections $[13,14]$ - to induce the development of VBNC cells in in vitro biofilms. Total live cells were detected by the developed qPCR protocol proven to reliably detect all viable (i.e., culturable and non-culturable) P. aeruginosa cells [8].

\section{Results}

\subsection{Assessment of Antibiotic Susceptibility}

The susceptibility of P. aeruginosa strains PAO1-N and C24 to ciprofloxacin and tobramycin was evaluated by minimal inhibitory concentration (MIC) determination. Strain PAO1-N was susceptible to both antibiotics, with MIC values of $0.25 \mathrm{mg} / \mathrm{L}$ and $1 \mathrm{mg} / \mathrm{L}$, respectively, whereas strain C24 showed low-level resistance with MIC values of 4 and $32 \mathrm{mg} / \mathrm{L}$, respectively.

\subsection{Stress Exposure of P. aeruginosa Biofilms and VBNC Cells Detection}

PAO1-N and C24 biofilms were developed in vitro in rich medium and then starved by immersion in non-nutrient medium $(\mathrm{NN})$, in $\mathrm{NN}+1 / 4 \mathrm{MIC}$ ciprofloxacin $(0.062 \mathrm{mg} / \mathrm{L}$ and $1 \mathrm{mg} / \mathrm{L}$, respectively) or in $\mathrm{NN}+1 / 4 \mathrm{MIC}$ tobramycin $(0.25 \mathrm{mg} / \mathrm{L}$ and $8 \mathrm{mg} / \mathrm{L}$, respectively). Biofilms were monitored for bacterial viability (qPCR counts) and culturability (colony forming unit (CFU) counts) for 170 days at approximately 15 -day intervals, starting 60 days from stress exposure. More than $92 \%$ of the biofilm matrix of both P. aeruginosa strains was consistently recovered, demonstrating the reliability of biofilm detachment from the petri dishes. Moreover, to confirm the reliability of qPCR in the detection of viable cells only, P. aeruginosa PAO1-N total viable cell (TVC) counts were performed by both qPCR and live/dead flow cytometry (FC) assays (Figure 1); similar live bacterial counts were always obtained, confirming the ability of the qPCR protocol to exclude extracellular DNA (eDNA) (Figure S1). 


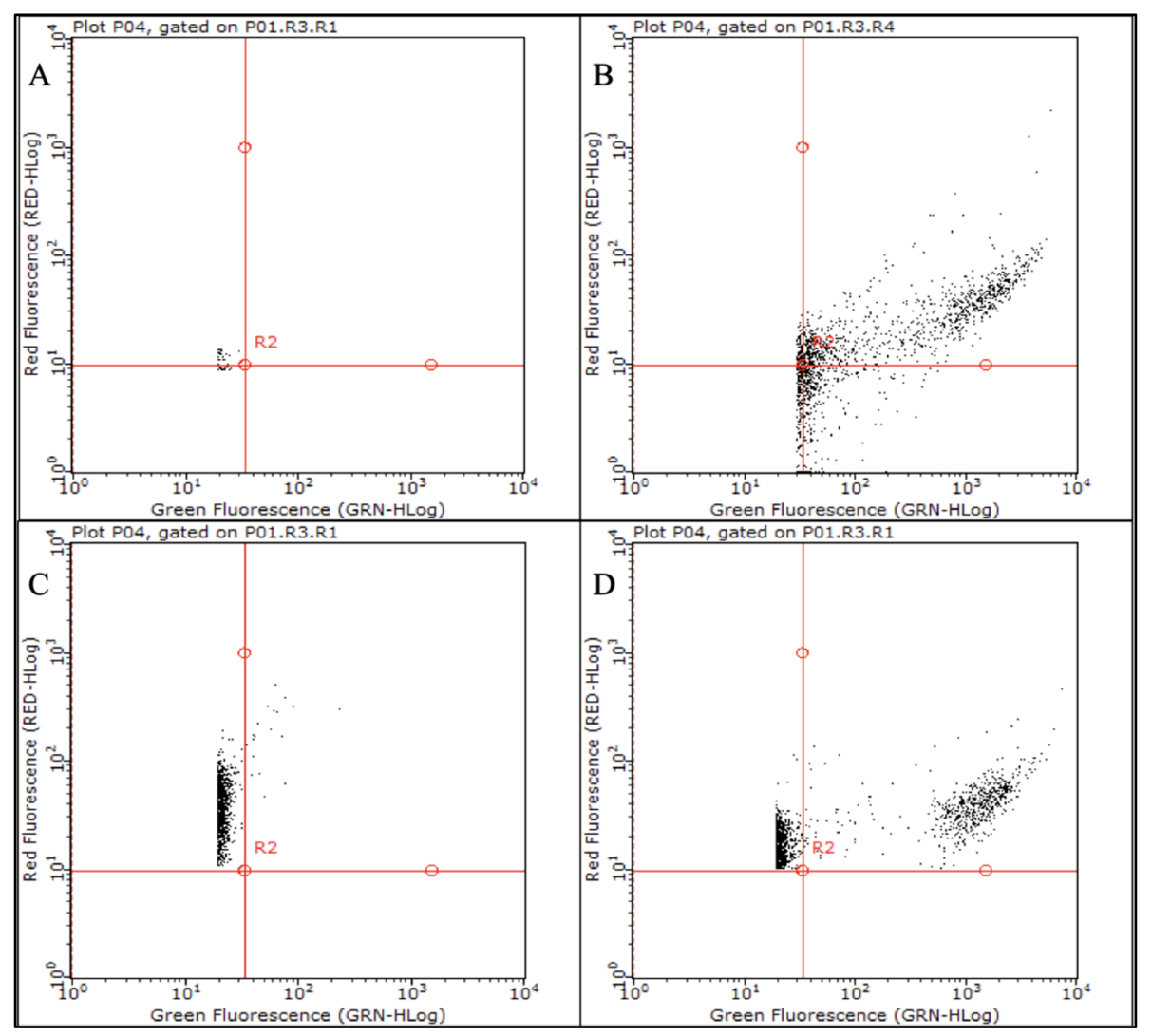

Figure 1. Analysis of a Pseudomonas aeruginosa PAO1-N biofilm by flow cytometry. The biofilm was analyzed after 120-day exposure to non-nutrient (NN) + tobramycin to determine its content in total viable cells, either undiluted or diluted 1:1000 in PBS. (A) PBS, negative control; (B) SYBR green-stained diluted biofilm; (C) Propidium iodide-stained diluted biofilm; (D) Live/dead-stained diluted biofilm. Viable cells were SYBR green- and live/dead-stained, whereas dead cells were propidium-iodide-stained.

Culturable cells of both strains were recovered at all time points. However, the two strains showed different amounts of TVCs and culturable cell counts over time (Figure 2).

Strain C24 showed a progressively lower number of both TVCs and culturable cells compared to the counts of day 0 , i.e., immediately before stress exposure. A significant difference between total viable and culturable cells (reflecting the presence of VBNC forms) was detected only in antibiotic-exposed biofilms, with a marked difference between ciprofloxacin- and tobramycin-exposed biofilms. In fact, the former biofilms contained a considerable amount (about $5 \times 10^{6}$ ) of VBNC cells, which, however, were no longer detectable at the end of the experiment, whereas in the latter biofilms, VBNC forms were consistently observed until the end of the experiment.

A large amount of VBNC forms, ranging from $85 \%$ to $97 \%$ of TVCs (Table 1), were counted in all $P$. aeruginosa PAO1-N biofilms since the first time point (60 days from exposure). The same amount of TVCs was detected over time in all experimental conditions. In biofilms exposed to NN (Figure 2A) and $\mathrm{NN}$ supplemented with ciprofloxacin (NN + CIP, Figure 2B), the number of culturable cells was stable until T120; this was followed by a constant increase in culturable cells until the end of the experiment, when VBNC cells were about 70\%. In tobramycin-exposed (NN + TOB) PAO1-N biofilms, the number of culturable cells was substantially stable up to T135, then it increased slightly; at the end of the experiment, VBNC cells were $97 \%$ of TVCs (Figure 2C).These findings indicate the development, in all test conditions, of a VBNC population (calculated as the difference between TVCs and culturable cells), which shrank gradually until the end of the experiment in PAO1-N NN and NN + CIP biofilms-likely due to the replication of culturable cells-whereas in NN + TOB biofilms, it remained fairly stable. 


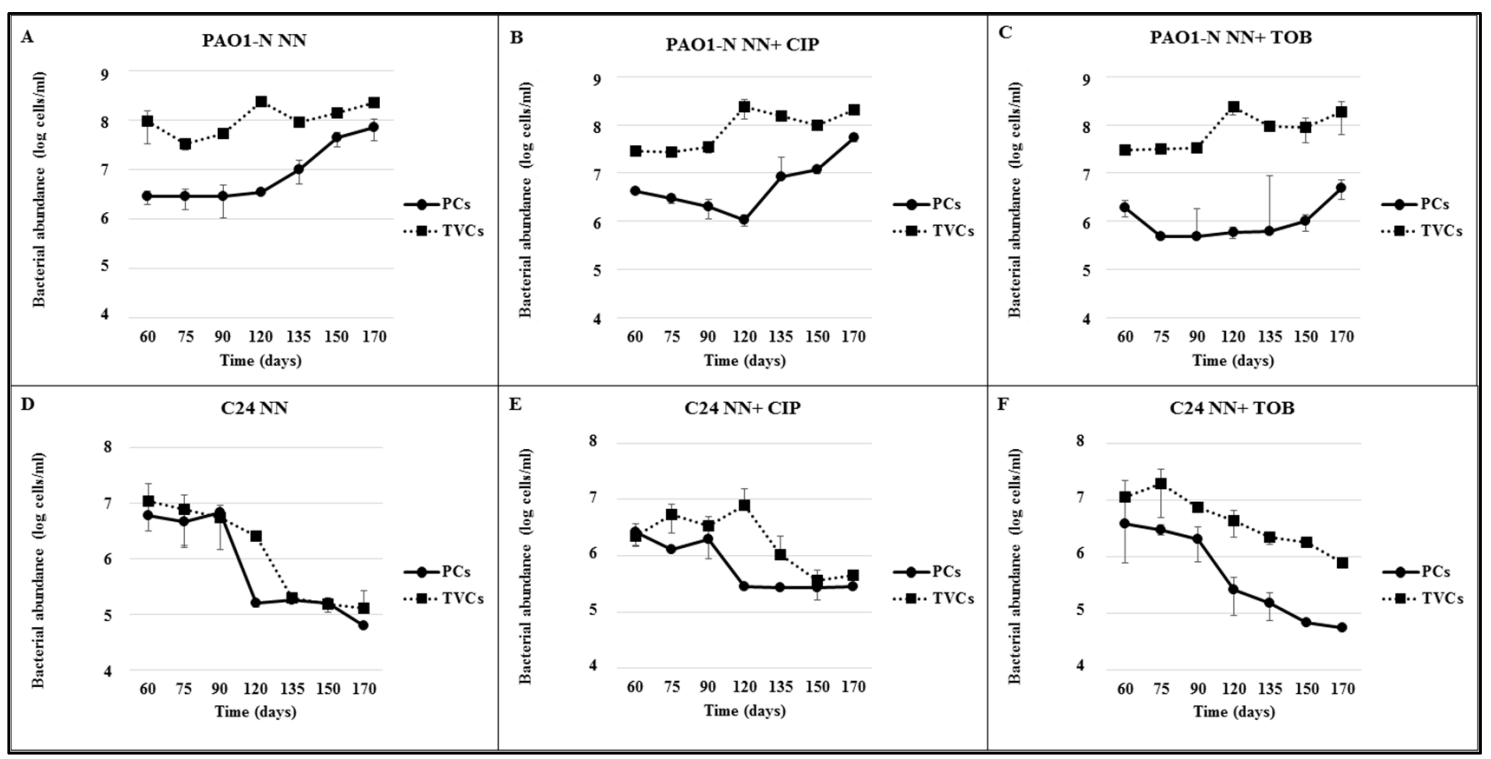

Figure 2. Viable cell counts determined in P. aeruginosa PAO1-N and C24 biofilms at different time intervals from stress exposure. Biofilms of P. aeruginosa PAO1-N and C24 were developed in vitro for $48 \mathrm{~h}$ in rich medium and subcultured in: (A,D) absence of nutrients (NN); (B,E) NN supplemented with CIP (NN + CIP); and (C,F) NN supplemented with TOB (NN + TOB). Total viable cells (TVCs, calculated as the average of qPCR and flow cytometry counts) and culturable cell counts at T0 were $1-2 \times 10^{9} / \mathrm{mL}(\mathrm{C} 24)$ and $1-4 \times 10^{9} / \mathrm{mL}$ (PAO1-N). Solid circles: culturable cells, detected by plate counts (PCs); solid squares: TVCs, culturable and non-culturable cells determined by qPCR. All counts are the average of three biological replicates \pm standard deviation. CIP, ciprofloxacin; TOB, tobramycin.

In contrast, at the first time point, $P$. aeruginosa $\mathrm{C} 24$ biofilms showed a narrow difference between culturable cells and TVCs, which was followed by a constant reduction in the formers in all test conditions until the end of the experiment. This trend was particularly marked in NN + TOB C24 biofilms (Figure 2F). In NN and NN + CIP C24 biofilms, culturable cells showed a marked reduction only between 90 and 120 days, whereas they remained unchanged from T120 until the end of the experiment (Figure 2D,E). Accordingly, NN and NN + CIP C24 biofilms did not show a stable VBNC population, whereas in NN + TOB C24 biofilms, a significant VBNC subpopulation was documented from T90 until the end of the experiment.

One further difference between the two strains was the appearance of specific colony phenotypes, observed throughout the experiment. Starting from T60, NN + TOB C24 biofilms subcultured on cystine lactose electrolyte deficient (CLED) agar produced slow-growing (i.e., not detectable before $72 \mathrm{~h}$ incubation), non-mucoid and non-swarming colonies, whereas NN C24 biofilms produced non-mucoid, fast-growing and swarming colonies. P. aeruginosa PAO1-N biofilms, starting from T120, produced a mucoid slow-growing phenotype in all test conditions when subcultured on CLED agar.

\subsection{Strain-and Antibiotic-Dependent Abundance of VBNC P. aeruginosa Cells}

The possible involvement of low-dose antibiotics in the induction of the VBNC phenotype [10] was tested by exposing PAO1-N and C24 biofilms to three stress conditions: starvation, starvation in the presence of 1/4 MIC CIP and starvation in the presence of 1/4 MIC TOB. The VBNC P. aeruginosa subpopulations detected in the three conditions were quantified and compared (Table 1).

A VBNC subpopulation was detected in all PAO1-N biofilms as early as 60 days from stress exposure at a frequency of $85 \%$ of TVCs (NN + CIP) or higher (NN and NN + TOB). However, after further 60 days (T120) their count fell to $67.7 \%$ in biofilms only starved and to $73.2 \%$ in biofilms exposed to $1 / 4$ MIC CIP, whereas in biofilms exposed to $1 / 4$ MIC TOB, the count actually increased to $97.2 \%$ (170 days). 
Table 1. VBNC P. aeruginosa abundance in the PAO1-N and C24 in vitro biofilms exposed to starvation alone or starvation in the presence of subinhibitory antibiotic concentrations.

\begin{tabular}{|c|c|c|c|c|}
\hline \multirow[t]{2}{*}{ Strain } & \multirow[t]{2}{*}{ Time (Days) } & \multicolumn{3}{|c|}{ TVCs (VBNC\%) } \\
\hline & & NN & $\mathrm{NN}+\mathrm{CIP}$ & $\mathrm{NN}+\mathrm{TOB}$ \\
\hline \multirow{7}{*}{ PAO1-N } & 60 & $9.51 \times 10^{7} \pm 0.13(97)$ & $2.85 \times 10^{7} \pm 0.23(85.1)$ & $2.98 \times 10^{7} \pm 0.14(93.4)$ \\
\hline & 75 & $3.41 \times 10^{7} \pm 0.84(91.6)$ & $2.76 \times 10^{7} \pm 0.48(89.1)$ & $3.18 \times 10^{7} \pm 0.50\left(98.5^{*}\right)$ \\
\hline & 90 & $5.39 \times 10^{7} \pm 1.06(91)$ & $3.46 \times 10^{7} \pm 0.80(94.2)$ & $3.29 \times 10^{7} \pm 0.04(98.5)$ \\
\hline & 120 & $2.45 \times 10^{8} \pm 0.56(98.6)$ & $2.37 \times 10^{8} \pm 0.06(99.5)$ & $2.32 \times 10^{8} \pm 0.75\left(99.7^{* *}\right)$ \\
\hline & 135 & $9.43 \times 10^{7} \pm 0.00(89)$ & $1.53 \times 10^{8} \pm 0.00(88)$ & $9.20 \times 10^{7} \pm 0.00\left(99.3^{*}\right)$ \\
\hline & 150 & $1.46 \times 10^{8} \pm 0.00(70)$ & $9.86 \times 10^{7} \pm 0.00\left(88.1^{* *}\right)$ & $8.98 \times 10^{7} \pm 1.73\left(98.8^{* *}\right)$ \\
\hline & 170 & $2.26 \times 10^{8} \pm 0.00(67.7)$ & $2.05 \times 10^{8} \pm 0.00(73.2)$ & $1.83 \times 10^{8} \pm 0.63\left(97.2^{* *}\right)$ \\
\hline \multirow{7}{*}{$\mathrm{C} 24$} & 60 & $1.07 \times 10^{7} \pm 0.09(44.8)$ & $2.21 \times 10^{6} \pm 0.73(\mathrm{ND})$ & $1.12 \times 10^{7} \pm 0.01(66.1)$ \\
\hline & 75 & $7.68 \times 10^{6} \pm 0.53(69.7)$ & $5.31 \times 10^{6} \pm 1.06(75.5)$ & $1.96 \times 10^{7} \pm 0.02\left(84.7^{* *}\right)$ \\
\hline & 90 & $5.35 \times 10^{6} \pm 0.00(\mathrm{ND})$ & $3.35 \times 10^{6} \pm 0.86(40.3)$ & $7.35 \times 10^{6} \pm 0.07\left(72.1^{* *}\right)$ \\
\hline & 120 & $2.49 \times 10^{6} \pm 0.30(93.8)$ & $7.85 \times 10^{6} \pm 0.45\left(96.4^{* *}\right)$ & $4.29 \times 10^{6} \pm 2.10\left(94^{* *}\right)$ \\
\hline & 135 & $1.93 \times 10^{5} \pm 0.31(\mathrm{ND})$ & $1.05 \times 10^{6} \pm 0.00\left(72.4^{* *}\right)$ & $2.21 \times 10^{6} \pm 0.55\left(93.2^{* *}\right)$ \\
\hline & 150 & $1.51 \times 10^{5} \pm 0.44(\mathrm{ND})$ & $3.58 \times 10^{5} \pm 0.79\left(23.2^{* *}\right)$ & $1.80 \times 10^{6} \pm 0.06\left(62.1^{* *}\right)$ \\
\hline & 170 & $1.27 \times 10^{5} \pm 0.06(51.1)$ & $4.57 \times 10^{5} \pm 0.33(38.7)$ & $7.84 \times 10^{5} \pm 0.61\left(93^{* *}\right)$ \\
\hline
\end{tabular}

${ }^{*} p \leq 0.05,{ }^{* *} p \leq 0.001 ; \mathrm{ND}$, not detectable; TVCs, total viable cells/mL expressed as average of three biological replicates \pm standard deviation.

At 60 days, VBNC cell abundance was lower in C24 than in PAO1-N biofilms, their highest frequency being $66.1 \%$, observed in NN + TOB biofilms. From this time on, the VBNC C 24 subpopulation in $\mathrm{NN}$ and $\mathrm{NN}+\mathrm{CIP}$ biofilms varied among time points, reaching respectively $51.1 \%$ and $38.7 \%$ of TVCs 170 days from stress exposure. In contrast, and similar to PAO1-N biofilm counts, C24 biofilms exposed to NN + TOB had a more stable ( $>60 \%$ ) VBNC subpopulation, which at 170 days reached $93 \%$ of TVCs.

\section{Discussion}

P. aeruginosa biofilms are the main cause of recurrent pulmonary infection in CF patients. Although several features of the infectious biofilm lifestyle, including signaling pathways $[10,15]$ and antibiotic resistance mechanisms [16], have largely been elucidated, little is known about the factors involved in the development of persistent and dormant bacterial forms. Persisters were initially described as bacterial forms which are produced stochastically and whose low metabolic activity is responsible for the failure of antibiotic treatment [17]; this is particularly critical in immunocompromised subjects such as CF patients [18]. More recently, a distinction between spontaneous and triggered persistence has been made [19]. The VBNC state is a later dormant state of persistence, characterized by the loss of the ability to grow on culture media; it can be induced by a variety of environmental stressors including starvation, excess light, temperature fluctuations and exposure to toxic compounds [5]. Low antibiotic concentrations can also contribute to the trigger of the VBNC state [11,12]. The present study was devised to establish whether starvation and subinhibitory drug concentrations-two typical features of the deepest biofilm layers [2,9]—induced VBNC forms in biofilms of a clinical (C24) and a laboratory (PAO1-N) strain of P. aeruginosa.

The ecf X-targeting qPCR used in this work has been previously described to accurately quantify TVCs due to the ability of the DNA extraction protocol to exclude eDNA [8]. The present work further supports the reliability of this procedure since P. aeruginosa counts obtained by qPCR resulted comparable to the amount of viable cells detected by flow cytometry (Figure S1).

VBNC cells of both P. aeruginosa strains were detected in all experimental conditions, in line with our previous study on S. aureus biofilms [11] and with the notion that the loss of culturability may be a survival strategy triggered by adverse environmental conditions, including starvation and toxic compounds [20-22]. 
Balaban et al. [19] have made a distinction between spontaneous and triggered persistence and indicated starvation as one of the most common triggers; they reported that when the trigger is removed, the persistent phenotype may be retained [19,23]. VBNC cells possess these features, although the factors that are required for the recovery of culturability are still unclear [11,21]. These data suggest that the VBNC state is a more advanced and less metabolically active state of triggered persisters and agree with the reports describing VBNC cells as the main obstacle to infection eradication [5]. Notably, after culture without nutrients for two months, all viable $P$. aeruginosa cells, either culturable and non-culturable, are to be considered as persisters. However, exposure of culturable persisters to a nutrient-rich environment can give rise to an antibiotic-susceptible subpopulation with full metabolic activity, whereas VBNC cells require the presence of specific resuscitation factors [21]. At variance with $S$. aureus biofilms exposed to vancomycin or quinupristin/dalfopristin [11], which uniformly lost culturability, a subset of culturable cells was detected in all P. aeruginosa biofilms. This different behavior may be explained by the greater stress-response ability of the opportunistic pathogen P. aeruginosa, which is endowed with a larger genome encoding several metabolic pathways and regulation systems to face the adverse environmental conditions. Among these, the lack of nutrients may be overcome via autophagy, with a small bacterial population feeding on lysed kin cells [24] and, after proliferation, restoring the original cell abundance.

Although VBNC cells were detected in both $P$. aeruginosa strains in all conditions, they were more numerous in biofilms exposed to subinhibitory tobramycin concentrations, as highlighted by their much greater prevalence in the tobramycin-exposed biofilms of both strains starting from T135 (Table 1). Subinhibitory tobramycin seems to hinder reversion to the culturable state or replication of the culturable subpopulation still found in the biofilm. This is suggestive of a hormetic response, i.e., a biphasic dose-response relationship (specifically, low-dose stimulation and high-dose inhibition) described by Davies and co-workers for various antibiotics, including aminoglycosides [10]. These results are also in agreement with those reported by Carvalhais et al., who described a role for protein synthesis inhibitors in the development of VBNC S. epidermidis [25], and by Lee and Bae for $P$. aeruginosa [12].

However, the two strains used in this study showed different behaviors. Whereas TVCs declined continuously in all C24 biofilms, their amount in tobramycin-exposed PAO1-N biofilms remained unchanged and slightly increased at the end of the experiment, always reaching greater values than those detected in C24 biofilms. This suggests a strain-specific behavior reflecting the greater ability of the lab strain to adjust to unfavorable conditions compared to the CF strain, which is used to living in symbiosis with the host. The more limited viability of the latter strain could be explained either by the maintenance in a non-symbiotic condition (in vitro biofilms) or by the loss of genetic information due to adaptation to the lung environment. Moreover, the mucoid shift detected in $P$. aeruginosa PAO1-N after stress exposure for 120 days is likely capable of protecting the bacterial cells, thus favoring strain survival.

\section{Materials and Methods}

\subsection{Bacterial Strains, Growth Media and Chemicals}

The extensively drug-resistant strain P. aeruginosa C24, isolated from a sputum sample of a chronic CF patient, and the reference strain P. aeruginosa PAO1-N, kindly provided by Prof. Paul Williams (Centre of Biomolecular Sciences, University of Nottingham, Nottingham, UK), were used as models. $P$. aeruginosa ATCC 27853, from the strain collection of the Microbiology section of the DiSVA (Polytechnic University of Marche, Ancona, Italy), was the reference strain in antibiotic susceptibility tests. All strains were cultured in lysogenic broth (LB) or CLED agar plates (all from Oxoid, Thermo Fisher Scientific, Waltham, MA, USA). Ciprofloxacin and tobramycin were purchased from Sigma-Aldrich (Saint Louis, MO, USA). 


\subsection{Antibiotic Susceptibility Test}

The MIC of ciprofloxacin and tobramycin was determined by the broth microdilution method according to CLSI guidelines [26] using P. aeruginosa ATCC 27853 as the reference strain.

\subsection{In Vitro P. aeruginosa Biofilm Development and Monitoring}

Biofilm models were developed in $35 \mathrm{~mm}$ petri dishes as described previously [27], with some modifications. Briefly, $2 \mathrm{~mL}$ aliquots of exponential $P$. aeruginosa cultures grown in LB broth were diluted to an optical density (OD) of $600=0.1$, inoculated into $35 \mathrm{~mm}$ petri dishes and grown at $37^{\circ} \mathrm{C}$ for $48 \mathrm{~h}$, refreshing the medium after the first $24 \mathrm{~h}$. Next, the medium was discarded and replaced with $3 \mathrm{~mL}$ of NN broth (M9 minimal medium without glucose) [11], alone or supplemented with 1/4 MIC of ciprofloxacin or tobramycin. This antibiotic concentration was tentatively chosen with reference to that used for S. aureus by Pasquaroli et al. [28]. The NN medium without/with antibiotic was refreshed every week. The abundance of culturable and TVCs, i.e., culturable and non-culturable, was assessed at 60,75, 90,120,135, 150 and 170 days, considering the first day of incubation in NN broth (starvation) as time (T) 0 . VBNC cells abundance was calculated as the difference between TVCs and culturable cells. At each time point, 3 biofilms per experimental condition were mechanically detached, resuspended in $1 \mathrm{~mL}$ phosphate buffered saline (PBS) and serially diluted 10-fold in PBS. Suitable dilutions were used in subsequent analyses.

\subsection{Biofilm Quantification}

Biofilm production and removal was measured in both $P$. aeruginosa strains by crystal violet staining as described previously [29], with some modifications. Briefly, P. aeruginosa biofilms were developed in $35 \mathrm{~mm}$ petri dishes in LB broth. After overnight incubation at $37^{\circ} \mathrm{C}$, the planktonic phase was removed, and its OD was recorded at $600 \mathrm{~nm}$. Biofilms were mechanically detached; after removal, the matrix was quantified by optical density $\left(\mathrm{OD}_{600}\right)$. The sessile phase was washed with sterile deionized water (DW) and stained with $1 \%$ crystal violet for $15 \mathrm{~min}$. After removing the dye, biofilms were washed with DW, resuspended in $96 \%$ ethanol and quantified by measuring OD at 570 $\mathrm{nm}$. Biofilm production was normalized by calculating the $\mathrm{OD}_{570} / \mathrm{OD}_{600}$ ratio. These tests were run in triplicate; each strain was tested for the amount of biofilm production and detachment.

\subsection{P. aeruginosa CFUs, $q P C R$ and Flow Cytometry Counts}

The abundance of total viable and culturable P. aeruginosa cells was determined by a species-specific qPCR and plate count, respectively.

For plate counts, serial dilutions of $P$. aeruginosa biofilms were plated on CLED agar; the abundance of CFUs was assessed after incubation for 24,48 , and $72 \mathrm{~h}$ at $37^{\circ} \mathrm{C}$.

For qPCR counts, 1:100 dilutions of 1 mL-PBS-resuspended P. aeruginosa biofilms were treated with DNase (reported to result as effective as propidium-monoazide in excluding the interference of the eDNA by Reyneke and colleagues [30]) and total DNA was extracted using QiAamp DNA Mini Kit (Qiagen, Hilden, Germany). Two $\mu \mathrm{L}$ of DNA were then amplified using the previously developed ecfX-targeting qPCR [8].

qPCR counts were validated by FC assays after live/dead staining using 1 X SYBR green and 40 $\mathrm{mg} / \mathrm{L}$ propidium iodide [31]. Assays were performed in a Guava Millipore cytometer using $200 \mu \mathrm{L}$ of a 1:1000 dilution of $P$. aeruginosa biofilms and analyzed by GUAVASOFT 2.2.3 software. Side scatter and green (GRN) fluorescence were gated to discriminate bacterial cells from the background; both channels were used at $488 \mathrm{~nm}$ and a threshold value was set in the GRN channel. SYBR green and propidium iodide were excited using a $488 \mathrm{~nm}$ laser, and the emissions were collected at 525/30 and $617 / 30 \mathrm{~nm}$, respectively. Signal detection was enhanced by logarithmic amplification (4 decades); to increase statistical significance, the total number of particles analyzed was set at 20,000 events/replicate. All assays were run in technical duplicate and biological triplicate. 
A difference $\geq 0.5 \log$ between plate and $\mathrm{qPCR} / \mathrm{FC}$ counts was held to indicate the presence of a VBNC P. aeruginosa subpopulation [8].

\subsection{Statistical Analysis}

The significance of the difference between qPCR and plate counts was assessed by Student's t-test (threshold, 0.05).

\section{Conclusions}

In conclusion, our findings highlight a strain-specific response to stress conditions and suggest that tobramycin, which is routinely used to treat CF lung infection, might contribute to the induction of VBNC P. aeruginosa forms in the lung of CF patients, likely through its ribosome binding-ability, which might trigger bacterial gene pathways involved in the development of the non-culturable phenotype [32].

They also stress the need for the routine adoption of a non-cultural microbiological approach to the diagnosis of P. aeruginosa pulmonary infection and confirm the usefulness of qPCR [8,33]; moreover, they suggest that the development of a species-specific FC protocol could constitute an alternative routine diagnostic technique.

The involvement of tobramycin in VBNC cells abundance in P. aeruginosa biofilms warrants further investigations, including a greater number of strains and the behavior of other drugs, particularly those included in the therapeutic protocols for CF P. aeruginosa lung infection.

Supplementary Materials: The following are available online at http://www.mdpi.com/2079-6382/9/7/399/s1, Figure S1: Viable P. aeruginosa quantifications by qPCR and flow cytometry. The amount of total viable P. aeruginosa PAO1-N cells was determined in in vitro biofilms exposed to A) starvation (NN), alone or B) coupled with 1/4 MIC ciprofloxacin $(\mathrm{NN}+\mathrm{CIP})$ or $\mathrm{C}$ ) tobramycin $(\mathrm{NN}+\mathrm{TOB})$, by ecfX-targeting qPCR and flow cytometry (FC) after live/dead staining. The bacterial counts obtained with the two techniques were compared. Results are reported as the average of three biological replicates \pm standard deviation.

Author Contributions: Conceptualization, F.B.; formal analysis, C.M.; funding acquisition, F.B.; investigation, G.M., N.C. and S.V.; methodology, G.M., S.V., B.C. and D.B.; project administration, F.B.; supervision, F.B.; validation, B.C., R.G., E.L., G.M., D.B. and F.B.; writing—original draft, G.M.; writing-review and editing, F.B. G.M. designed the experimental plan, developed the biofilm model, performed the qPCR assays and interpreted the obtained results. N.C. performed the microbiological and qPCR assays, evaluating the bacterial viability and culturability. S.V. developed the flow cytometry protocol and performed the related assays. B.C. contributed to the experimental design and critically commented on the obtained results. R.G. critically commented on the experimental data, focusing on the antibiotics' action on the microbial cells. E.L. contributed to the experimental design and interpretation. G.M. contributed to the experimental interpretation. C.M. performed the statistical analysis on the obtained results. D.B. contributed to the flow cytometry protocol development and data interpretation. F.B. coordinated the whole experiment and interpreted the obtained data in the context of antibiotic tolerance and persistence. All authors have read and agreed to the published version of the manuscript.

Funding: This research was funded by Italian Cystic Fibrosis Foundation, grant number FFC\#13/2017 and by Università Politecnica delle Marche, grant number PSA 2016. The APC was funded by Italian Cystic Fibrosis Foundation, grant number FFC\#16/2019.

Acknowledgments: Part of these results have been presented at the "Biofilm 8" meeting, 27-29 May 2018, Aarhus University, Aarhus C, Denmark and at the "Microbiology 2019" meeting, XXXIII SIMGBM Congress, 19-22 June 2019, Università di Firenze, Firenze, Italy.

Conflicts of Interest: The authors declare no conflict of interest.

\section{References}

1. Hoo, Z.H.; Edenborough, F.P.; Curley, R.; Prtak, L.; Dewar, J.; Allenby, M.I.; Nightingale, J.A.; Wildman, M.J. Understanding Pseudomonas status among adults with cystic fibrosis: A real-world comparison of the Leeds criteria against clinicians' decision. Eur. J. Clin. Microbiol. Infect. Dis. 2018, 37, 735-743. [CrossRef] [PubMed]

2. Lee, K.; Yoon, S.S. Pseudomonas aeruginosa Biofilm, a Programmed Bacterial Life for Fitness. J. Microbiol. Biotechnol. 2017, 27, 1053-1064. [CrossRef] [PubMed] 
3. Linnane, B.; Kearse, L.; O'Connell, N.H.; Fenton, J.; Kiernan, M.G.; Dunne, C.P. A case of failed eradication of cystic fibrosis-related sinus colonisation by Pseudomonas aeruginosa. BMC Pulm Med. 2015, 15, 114. [CrossRef] [PubMed]

4. Lewis, K. Persister cells: Molecular mechanisms related to antibiotic tolerance. Handb. Exp. Pharm. 2012, 211, 121-133.

5. Ayrapetyan, M.; Williams, T.C.; Oliver, J.D. Bridging the gap between viable but non-culturable and antibiotic persistent bacteria. Trends Microbiol. 2015, 23, 7-13. [CrossRef]

6. Deschaght, P.; Schelstraete, P.; Van Simaey, L.; Vanderkercken, M.; Raman, A.; Mahieu, L.; Van Daele, S.; De Baets, F.; Vaneechoutte, M. Is the improvement of CF patients, hospitalized for pulmonary exacerbation, correlated to a decrease in bacterial load? PLOS ONE 2013, 8, e79010. [CrossRef]

7. Le Gall, F.; Le Berre, R.; Rosec, S.; Hardy, J.; Gouriou, S.; Boisramé-Gastrin, S.; Vallet, S.; Rault, G.; Payan, C.; Héry-Arnaud, G. Proposal of a quantitative PCR-based protocol for an optimal Pseudomonas aeruginosa detection in patients with cystic fibrosis. BMC Microbiol. 2013, 13, 143. [CrossRef]

8. Mangiaterra, G.; Amiri, M.; Di Cesare, A.; Pasquaroli, S.; Manso, E.; Cirilli, N.; Citterio, B.; Vignaroli, C.; Biavasco, F. Detection of viable but non-culturable Pseudomonas aeruginosa in cystic fibrosis by qPCR: A validation study. BMC Infect. Dis. 2018, 18, 701. [CrossRef]

9. Olivares, E.; Badel-Berchoux, S.; Provot, C.; Prévost, G.; Bernardi, T.; Jehlet, F. Clinical Impact of Antibiotics for the Treatment of Pseudomonas aeruginosa Biofilm Infections. Front. Microbiol. 2020, 10, 2894. [CrossRef]

10. Davies, J.; Spiegelman, G.B.; Yim, G. The world of subinhibitory antibiotic concentrations. Curr. Opin. Microbiol. 2006, 9, 445-453. [CrossRef]

11. Pasquaroli, S.; Zandri, G.; Vignaroli, C.; Vuotto, C.; Donelli, G.; Biavasco, F. Antibiotic pressure can induce the viable but non-culturable state in Staphylococcus aureus growing in biofilms. J. Antimicrob. Chemother. 2013, 68, 1812-1817. [CrossRef] [PubMed]

12. Lee, S.; Bae, S. Molecular viability testing of viable but non-culturable bacteria induced by antibiotic exposure. Microb. Biotechnol. 2018, 11, 1008-1016. [CrossRef] [PubMed]

13. Stanojevic, S.; Waters, V.; Mathew, J.L.; Taylor, L.; Ratjen, F. Effectiveness of inhaled tobramycin in eradicating Pseudomonas aeruginosa in children with cystic fibrosis. J. Cyst. Fibros. 2014, 13, 172-178. [CrossRef]

14. Al-Obaidi, H.; Kalgudi, R.; Zariwala, M.G. Fabrication of inhaled hybrid silver/ciprofloxacin nanoparticles with synergetic effect against Pseudomonas Aeruginosa. Eur J. Pharm Biopharm. 2018, 128, 27-35. [CrossRef]

15. Lee, J.; Zhang, L. The hierarchy quorum sensing network in Pseudomonas Aeruginosa. Protein Cell. 2015, 6, 26-41. [CrossRef] [PubMed]

16. Soto, S.M. Role of efflux pumps in the antibiotic resistance of bacteria embedded in a biofilm. Virulence 2013, 4, 223-229. [CrossRef]

17. Lewis, K. Persister cells, dormancy and infectious disease. Nat. Rev. Microbiol. 2007, 5, 48-56. [CrossRef]

18. Mulcahy, L.R.; Burns, J.L.; Lory, S.; Lewis, K. Emergence of Pseudomonas aeruginosa strains producing high levels of persister cells in patients with cystic fibrosis. J. Bacteriol. 2010, 192, 6191-6199. [CrossRef]

19. Balaban, N.Q.; Helaine, S.; Lewis, K.; Ackermann, M.; Aldridge, B.; Andersson, D.I.; Brynildsen, M.P.; Bumann, D.; Camilli, A.; Collins, J.J.; et al. Definition of guidelines for research on antibiotic persistence. Nat. Rev. Microbiol. 2019, 17, 441-448. [CrossRef]

20. Balaban, N.Q.; Merrin, J.; Chait, R.; Kowalik, L.; Leibler, S. Bacterial persistence as a phenotypic switch. Sci. Cit. Index 2004, 305, 1622-1625. [CrossRef]

21. Li, L.; Mendis, N.; Trigui, H.; Oliver, J.D.; Faucher, S.P. The importance of the viable but non-culturable state in human bacterial pathogens. Front. Microbiol. 2014, 5, 258. [CrossRef] [PubMed]

22. Ayrapetyan, M.; Williams, T.C.; Baxter, R.; Oliver, J.D. Viable but nonculturable and persister cells coexist stochastically and are induced by human serum. Infect. Immun. 2015, 83, 4194-4203. [CrossRef] [PubMed]

23. Moradali, M.F.; Ghods, S.; Rehm, B.H. Pseudomonas aeruginosa Lifestyle: A paradigm for adaptation, survival, and persistence. Front. Cell Infect. Microbiol. 2017, 7, 39. [CrossRef] [PubMed]

24. Turnbull, L.; Toyofuku, M.; Hynen, A.L.; Kurosawa, M.; Pessi, G.; Petty, N.K.; Osvath, S.R.; Cárcamo-Oyarce, G.; Gloag, E.S.; Shimoni, R.; et al. Explosive cell lysis as a mechanism for the biogenesis of bacterial membrane vesicles and biofilms. Nat. Commun. 2016, 7, 11220. [CrossRef] [PubMed]

25. Carvalhais, V.; Pérez-Cabezas, B.; Oliveira, C.; Vitorino, R.; Vilanova, M.; Cerca, N. Tetracycline and rifampicin induced a viable nonculturable state in Staphylococcus epidermidis biofilms. Future Microbiol. 2018, 13, 27-36. [CrossRef] [PubMed] 
26. Clinical and Laboratory Standards Institute. Performance Standards for Antimicrobial Susceptibility Testing: Twenty-seventh Informational Supplement M100-S27; CLSI: Wayne, PA, USA, 2017.

27. Sabaeifard, P.; Abdi-Ali, A.; Soudi, M.R.; Dinarvand, R. Optimization of tetrazolium salt assay for Pseudomonas aeruginosa biofilm using microtiter plate method. J. Microbiol. Methods. 2014, 105, 134-140. [CrossRef]

28. Pasquaroli, S.; Citterio, B.; Mangiaterra, G.; Biavasco, F.; Vignaroli, C. Influence of Sublethal Concentrations of Vancomycin and Quinupristin/Dalfopristin on the Persistence of Viable but Non-Culturable Staphylococcus aureus Growing in Biofilms. J. Antimicrob. Chemother. 2018, 73, 3526-3529. [CrossRef]

29. Camilli, R.; Pantosti, A.; Baldassarri, L. Contribution of serotype and genetic background to biofilm formation by Streptococcus pneumoniae. Eur. J. Clin. Microbiol. Infect. Dis. 2011, 30, 97-102. [CrossRef]

30. Reyneke, B.; Ndlovu, T.; Khan, S.; Khan, W. Comparison of EMA-, PMA- and DNase qPCR for the determination of microbial cell viability. Appl Microbiol. Biotechnol. 2017, 101, 7371-7383. [CrossRef]

31. Zandri, G.; Pasquaroli, S.; Vignaroli, C.; Talevi, S.; Manso, E.; Donelli, G.; Biavasco, F. Detection of viable but non-culturable staphylococci in biofilms from central venous catheters negative on standard microbiological assays. Clin. Microbiol. Infect. 2012, 18, E259-E261. [CrossRef]

32. Wood, T.K.; Song, S.; Yamasaki, R. Ribosome dependence of persister cell formation and resuscitation. J. Microbiol. 2019, 57, 213-219. [CrossRef] [PubMed]

33. Héry-Arnaud, G.; Nowak, E.; Caillon, J.; David, V.; Dirou, A.; Revert, K.; Munck, M.R.; Frachon, I.; Haloun, A.; Horeau-Langlard, D.; et al. Evaluation of quantitative PCR for early diagnosis of Pseudomonas aeruginosa infection in cystic fibrosis: A prospective cohort study. Clin. Microbiol. Infect. 2017, 23, 203-207. [CrossRef] [PubMed]

Sample Availability: Samples of the bacterial strains are available from the authors.

(C) 2020 by the authors. Licensee MDPI, Basel, Switzerland. This article is an open access article distributed under the terms and conditions of the Creative Commons Attribution (CC BY) license (http://creativecommons.org/licenses/by/4.0/). 